\title{
Robots in Cancer Surgery: A Boon or Bane
}

\author{
Yusuf Jamal1, Abdulrahman M. Alshahrani2, Jamal M. Arif ${ }^{3}$, Feras M. Almarshad2* \\ ${ }^{1}$ Faculty of Medicine, Ternopil National Medical University, Ternopil, Ukraine \\ ${ }^{2}$ Department of Internal Medicine, College of Medicine, Shaqra University, Shaqra, KSA \\ ${ }^{3}$ Department of Biochemistry, College of Medicine, Shaqra University, Shaqra, KSA \\ Email: ^falmarshad@su.edu.sa
}

How to cite this paper: Jamal, Y., Alshahrani, A.M., Arif, J.M. and Almarshad, F.M. (2020) Robots in Cancer Surgery: A Boon or Bane. Journal of Cancer Therapy, 11, 803-823.

https://doi.org/10.4236/jct.2020.1112070

Received: November 8, 2020

Accepted: December 12, 2020

Published: December 15, 2020

Copyright $\odot 2020$ by author(s) and Scientific Research Publishing Inc. This work is licensed under the Creative Commons Attribution International License (CC BY 4.0).

http://creativecommons.org/licenses/by/4.0/

\begin{abstract}
It is an ongoing task to keep exploring and applying the best available technology to alleviate the pain and sufferings of the cancer patients. Since the discovery of robotic surgery, da Vinci surgical systems have played a special and significant role in cancer surgeries worldwide, however, surgeons are still skeptical with the clinical and oncological outcomes which are almost comparable to the laparoscopic approach in several cancers. Many meta-analyses using mostly retrospective studies indicated significant advantage of robotic surgery over laparoscopic or open surgery approaches for various cancers, however, scarcity of technically sound robot savvy surgeons and quality multicentered, multinational, coordinated, random clinical trials had not done justice to the positives of robotic surgery which were quite often suppressed by the negative factors like operative cost and oncological outcomes. Nevertheless, robotic surgery approach has been clinically accepted for hysterectomy and prostatectomy. This overview briefly discusses the comparative approaches (open, laparoscopic, robotic assisted) and their clinical outcomes in the surgery of various cancers.
\end{abstract}

\section{Keywords}

Da Vinci, Robotic Surgery, Laparoscopic Surgery, Cancers, Oncologic Outcomes

\section{Introduction}

Traditional Surgery (TS) where surgeons make large incisions on the body of the patient and perform surgery using surgical instruments and a certified procedure to investigate or treat a pathological condition is still practiced for most clinical cases. This was transformed later into Minimally Invasive Surgery (MIS), a practice of performing surgery through small incisions using specialized sur- 
gical instruments assisted by a laparoscopic camera for various surgeries. Contrary to the TS, the reduced incision size, less wound trauma, discomfort, recovery time and cost in MIS received tremendous popularity within the surgical community towards these techniques. At the same time, MIS is technically difficult and poses the risk of surgical complications mainly due to lack of precision and availability of instrument technology. While finding solutions as to how surgical precision can be attained, many researchers started finding a way to use robots to assist surgeons in performing surgeries.

In the past three decades, there have been many different versions and types of robots in performing Robotic Surgery (RS) that were introduced and applied for surgery of various cancers and other types of surgeries. In this review, we will discuss the development and transformation journey of various robotic systems mainly da Vinci robots and their significance in the surgery of a few prominent cancers.

\section{Method}

This systematic review includes selective search for relevant original articles and reviews, case reports, randomized and non-randomized controlled trials, cohort studies from 2010-2020 using Pubmed and Google Scholar. We used the following keywords: history of robotic surgery, da Vinci robots, laparoscopic surgery, robotic surgery in lung, liver, colorectal, gastric, breast, prostate and gynecological cancer. Articles were restricted to English.

\section{Results}

Our search resulted in total of 918 publications and after the removal of duplicate studies by browsing the titles subsequently reduced the numbers to 300 (Figure 1). After assessing the abstracts for duplicate, and irrelevant data to our review scope, an additional 145 were excluded. Out of 120 full length articles, 10 were further excluded due to overlapping and inconclusive data. Thirty-nine references were replaced with the respective original references. Finally, 110 relevant studies were included in the final review.

\subsection{Development and Transformation of Robots}

RS dates to 1985 when Kwoh and colleagues performed neurosurgical biopsies with greater precision [1]. Three years later, Davies et al. [2] used a PUMA 560 to perform a transurethral resection of the prostate. Their success led to the development of the PROBOT which was designed specifically for transurethral resection of the prostate. However, almost simultaneously, the ROBODOC was being developed by Integrated Surgical Supplies Ltd. (USA), aiming to maneuver the femur in the hip replacement surgeries with greater precision [3] [4].

The Stanford Research Institute (SRI, USA) was joined by several scientists from the NASA-Ames team and they together developed a dexterous telemanipulator for hand surgery [5]. It offered the surgeon the impression of directly 


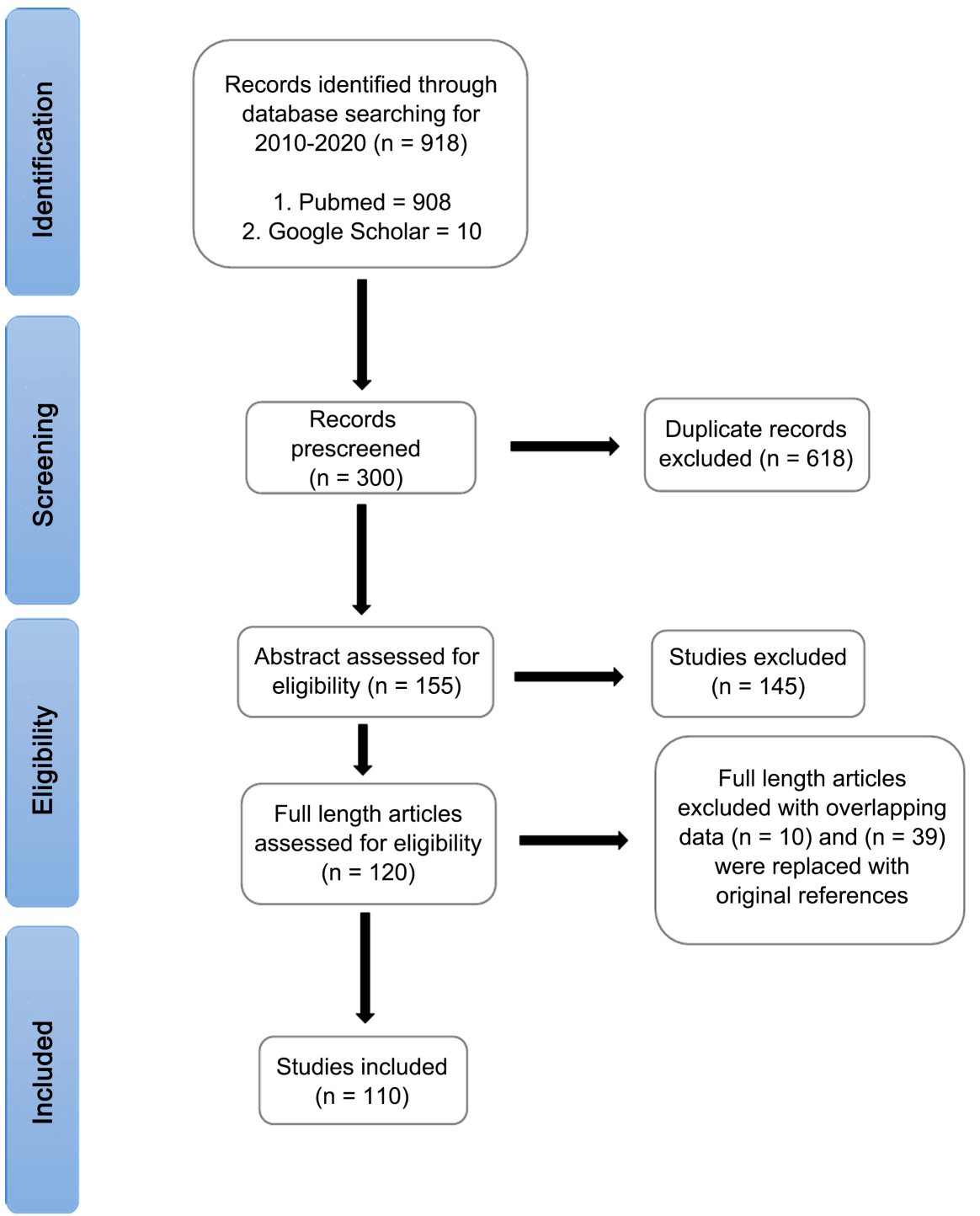

Figure 1. Preferred Reporting Items for Systematic Reviews and Meta-analysis (PRISMA) flow diagram for selection of relevant studies.

working on the patient, rather than across the bed. As these robots were being developed, the engineering team along with general surgeons and endoscopists recognized the potential of these devices in improving the limitations of traditional laparoscopic operations. The US Army later developed a system whereby a surgeon could operate a wounded soldier remotely hoping to reduce wartime casualties by precluding exsanguinating wounded soldiers until they entered the hospital. The device, however, has been successfully demonstrated on animal models but has yet to be applied for actual treatment of casualties on the battlefield [6] [7] [8] [9] [10].

\subsection{Types of Robotic Systems}

In the surgical arena, three major types of robotic systems are currently in operation i.e. active, semi-active, and master-slave. Active systems operate largely 
autonomously under the operative surgeon's control and execute pre-programmed tasks. PROBOT and ROBODOC platforms are good examples of the Active systems. Semi-active systems allow complementing the pre-programmed component of these robot systems with a component controlled by a surgeon. Lastly, formal master-slave systems lack any of the pre-programmed or autonomous components. They depend entirely on the operation of the surgeons. Hand movements of the surgeon are transmitted to laparoscopic surgical instruments that faithfully replicate the manual operation of the surgeon-but intracorporeally. The master-slave robot systems have been tested on many different forms of surgery.

In 1992, Computer Motion (USA) developed an Automated Endoscopic System for Optimal Positioning (AESOP), the first voice-controlled surgical robot to obtain USFDA approval for use in the operating room (OR) [4] [10]. Later, in 1996, Computer Motion unveiled HERMES with an integrated voice control and feedback into other OR components [9]. In the same year, Computer Motion quickly manufactured the first complete robotic surgery platform, ZEUS which had the same software and platform as the robotic arm of AESOP but was equipped with laparoscopic instrumentation [9]. Early models of ZEUS only had 6 degrees of freedom (DOFs) but later models were made equipped with 7 DOFs. In addition, the stabilization of motion and the removal of tremor were the main features of the latest ZEUS models [9]. ZEUS received the USFDA approval in 2001 [10].

Computer Motion originally targeted the cardiothoracic surgery for ZEUS, with a specific focus on creating a minimally invasive Coronary Artery Bypass Graft surgery (CABG) technique. However, robotic CABG procedures faced several difficulties including insufficient space to place instruments appropriately besides the effectiveness which was found to be comparable with the catheter procedures. Moreover, ZEUS also found a niche in urological surgery, but ZEUS was discontinued in 2003 following the merger of Computer Motion with its rival Intuitive Surgical (USA).

Later, Massachusetts Institute of Technology (MIT, USA) developed its first prototype, the Silver Falcon powered with the haptic interface device PHANToM [11]. The Silver Falcon had 7 DOFs: 3 inside the base positioner, 3 inside the wrist, plus the distal grasper. However, several drawbacks were observed in the Silver Falcon such as weak structural rigidity, inadequate grip strength to handle large needles, and counterbalancing gravitational compensation which was rectified in the next version, the Black Falcon, with an addition of another DOF to the wrist and detachable the end effector [11]. It had 8 DOF cable driven teleoperator slave which uses a detachable 4 DOF wrist plus a 1 DOF gripper integrated with a 3 DOF base positioner.

The Black Falcon was equipped with the force input from the surgical manipulator to the surgeon through PHANToM. It was later found out that the representation of force presented more barriers than support for certain activities, 
such as suturing. There were also some concerns about incorporating haptics into a clinical application system. The haptic technology was found to have visualizing deflection in soft tissues that occurred before force became a factor. However, the perception from the mirror and stereoscopic vision system compensated for the dismissal of force feedback [11] [12].

\subsection{Da Vinci Robotic Surgical Systems}

Da Vinci Surgical System developed by Intuitive Surgical (USA) was approved by FDA in 2000 for prostatectomies, cardiac valve repair and gynecological surgeries [13] [14]. Despite high cost and software issues, there are 5582 installed bases of da Vinci Surgical Systems worldwide till 2019-2020 including 3531 in USA, 977 in Europe, 780 in Asia and remaining 294 in the rest of the world [15]. Using da Vinci Surgical Systems, an estimated $>1.2$ million surgeries have been performed worldwide [15]. Da Vinci Surgical Systems have been applied in many surgical procedures such as gynecological (hysterectomy, sacrocolpopexy), urologic (prostatectomy, partial nephrectomy), cardiothoracic (thoracic surgery, mitral valve repair), hernia repair, colorectal, cholecystectomy, bariatric and transoral surgeries [15]. In the United States, da Vinci surgeries are the \#1 option chosen by women with gynecologic cancer while $86 \%$ with prostate cancer opted for prostatectomy in 2009 [13].

$\mathrm{Da}$ Vinci has four-generational platforms which include the first generation da Vinci standard Surgical System, second generation da Vinci S Surgical System, third generation da Vinci Si Surgical System and the fourth generation da Vinci X, Xi and SP Surgical Systems. Da Vinci Surgical Systems provide immersive 3DHD (3-dimensional high density) visualization, precise tremor-free endoscope control, advanced instruments of stapler and energy, scaled and tremor filtered instrument movement, improved surgeon ergonomics and multi-specialty surgical platforms [15].

Two exclusive features of da Vinci Xi device worth mentioning include an EndoWrist One Sealer tube and EndoWrist Stapler 45. The EndoWrist Sealer is a completely wristed instrument designed for sealing and cutting vessels up to 7 $\mathrm{mm}$ in bundles of diameter and tissue which fit in the instrument's jaws. While the EndoWrist Stapler is fully operated by the surgeon using da Vinci Xi System, receives wristed articulation and input from SmartClamp, which senses if the stapler jaws are tightly closed on the tissue before firing. The EndoWrist Stapler is intended for gynecological, general, urological and thoracic surgeries to resect, transect, and/or create anastomoses.

Further, da Vinci Xi System is designed to be compliant with the Firefly Fluorescence Imaging System of Intuitive Surgical. During minimally invasive procedures, the Firefly device allows surgeons to switch between normal, visible light and near-infrared imaging. When a surgeon uses the Firefly System in conjunction with an injectable fluorescent dye, tissue with blood flow is highlighted in green color, and tissue without blood flow appears gray in the 
surgeon's view. Moreover, da Vinci Surgical Systems provide an extraordinary seven DOF to have greater operational control for surgeons which is one of the best-selling features for da Vinci systems.

\subsection{Clinical Applications of Da Vinci Robots in Cancer Surgery}

The advancement of robotics has been implemented in various clinical instruments and its specific applications in different cancer surgeries and cardiothoracic surgery are noticeable. With several advantages like precision over the traditional surgeries, robotic surgeries have also shown certain disadvantages for various cancers and cardiac surgeries. Below, we have discussed the application of da Vinci robots in the surgery of some of the major cancers.

\subsubsection{Lung Cancer}

With the advent of minimally invasive Video-Assisted Thoracoscopic Surgery (VATS) for lung cancer, a reduction in postoperative discomfort, decreased cytokine responses, quicker resumption of daily activities, better esthetic and functional outcomes were reported when compared to the traditional thoracotomy [16]-[21]. Nevertheless, most thoracic surgeons have not approved VATS and approached thoracotomy for pulmonary lobectomy in lung cancer patients [22] Further, possible difficulties in performing a routine full mediastinal lymphadenectomy for lung cancer surgery, technological challenges and steep learning curve for VATS made it less favorable for the thoracic surgeons [23] [24].

Overcoming many shortcomings of VATS, da Vinci Xi robot-assisted surgery has provided many benefits including better 3DHD visualization and better ergonomics with greater freedom of movement [25]-[31]. In addition, as robot-assisted surgery and VATS are associated with quicker recovery than open surgery (OS). Minimally invasive resections of the lungs for early-stage lung cancer (stages I or II) with robot-assisted lung resection resulted in low bleeding conversion rate and low mortality thereby indicating it a safe and efficient robotic approach to locally advanced lung cancer [26]-[31].

Most of the comparative studies between thoracotomy, VATS and robot-assisted thoracic surgery (RATS) were done retrospectively and need for randomized controlled studies evaluating the different operations, modalities and extent of resection is still required [32]. Park et al. [33] reported using the State Inpatient Databases (2008-2010) to analyze and compare 33,095 patients who underwent lobectomy. The distribution of patients for the procedures was for open $(20,238)$, VATS $(12,427)$ and RATS $(430)$. In propensity-matched analysis, RATS showed significant reductions in mortality, length of stay, and overall complication rates when compared with open thoracotomy or VATS. However, when compared with VATS, these differences were not statistically significant [33].

\subsubsection{Liver Cancer}

Minimally invasive hepatobiliary surgery began in 1987 with the first laparos- 
copic cholecystectomy [34] [35]. Since the introduction of robotic liver resection, both minor and major operations such as hepatectomies and robotic-assisted hepatic interventions and liver donation for liver transplantation by living humans have been successfully performed [36] [37] [38]. Although the first series of robotic-assisted laparoscopic liver resections were reported by Giulianotti and colleagues [2003], almost every meta-analysis has rightly pointed towards the lack of randomized controlled trials to compare robotic with laparoscopic liver surgeries [36] [37] [38] [39]. However, Lafaro et al. [38] has recently reported comparative analysis of several high-quality nonrandomized as well as meta-analyses studies. A retrospective study while evaluating 97 patients who underwent robotic liver resections (13 major resections; 84 minor resections; 51 inferior segmentectomia; 33 posterosuperior segment ectomies) reported that $66 \%$ of the patients were discharged within 3 days of the robotic procedure [40]. However, hospital stays longer than 3 days predicted possible extent of resection, occurrence of complications and operative time greater than 3.5 hours [40].

Later, Nota and colleagues [41] performed a multinational, retrospective, study of 51 robotic and 145 open posterosuperior liver resections between 2009 and 2016. Propensity-matched analysis of 31 robotic and 31 open resections of posterosuperior segments showed no differences in median operative time, estimated blood loss, major complications rates or readmission rates.

Further, multinational, multicenter, retrospective study of robotic-assisted surgery for hepatocellular carcinoma, cholangiocarcinoma, or gallbladder cancer on the patients between 2006 and 2016 was conducted [42]. The study included 61 major and minor resections (56\% for hepatocellular carcinoma, $26 \%$ for cholangiocarcinoma, and $18 \%$ for gallbladder cancer) with a median follow-up time of 75 months achieved five-year overall survival of $56 \%$ of the patients [42].

Another meta-analysis with 938 patients (435 robotic hepatectomies vs 503 laparoscopic hepatectomies) performed by Guan and colleagues [43] examined 13 nonrandomized control studies, which included patients diagnosed with multiple types of liver lesions reported that the robotic hepatectomy group had decreased intraoperative blood loss by an average of $69.9 \mathrm{~mL}$. However, it also resulted in longer operative times otherwise there were no significant differences between the two procedures in the other perioperative outcomes.

Moreover, Fruscione et al. [44] ultimately reported improved outcomes with less frequent postoperative ICU admissions and decreased 90-day readmission rates from a recent large, single-center, retrospective analysis of 173 major minimally invasive hepatectomies (57 robotic-assisted vs 116 laparoscopic). Further, they showed no significant difference in blood loss, operative times, or length of stay between the two groups.

Expert laparoscopic surgery has similar outcomes to expert robotic surgery. However, the major advantages include ease of adoption, ergonomics, enabling minimally invasive surgery, safe, feasible and facilitating indocyanine green im- 
aging. However, robotic-assisted liver surgeries encountered with inability to palpate the liver, increased incision size for larger specimen removal, additional operative time to dock and undock, potential of argon gas vascular embolism and high cost. Nevertheless, large, multicenter, randomized and prospective clinical trials are needed to analyze the exact role of robotic liver surgery in patients with complex and malignant liver tumors and to formulate the insurance coverage policies for future application of the robotic surgery [36] [37] [38] [39].

\subsubsection{Colorectal Cancer}

Since the first laparoscopic colon resections in 1991 by several groups, has established that this approach is technically feasible [45] [46] [47], however, the safety of this approach remains a question for both the surgeons and patients. Several randomized controlled trials and a meta-analysis demonstrated decreased time to flatus, postoperative pain, and length of hospital stay for laparoscopic patients compared to the open colectomy [48] [49] [50] One of the major drawbacks of the laparoscopic approach is relatively higher conversion rate of $14.6 \%$ to open colectomy, largely due to its inability to visualize advanced disease or critical structures which increases morbidity, mortality, need of blood transfusion and length of hospital stay [51] [52] [53] [54].

Further, to overcome the inherent limitations in the laparoscopic surgical instruments, a switch to Robotic-assisted Laparoscopic Surgery (RALS) was made and several studies have shown it to be secure and feasible for different colorectal procedures. Unfortunately, relatively higher cost and the need of expert robotic surgeons have limited its application in the colorectal surgeries. A nonrandomized prospective study with 378 patients having stage I-III colorectal cancer who underwent the robotic or laparoscopic resection showed statistically no significant difference in the number of nodes or locoregional recurrence rates between the robotic and laparoscopic groups [55]. Similar results were also reported in a comparative study using open, laparoscopic, and robotic approaches for right-sided colon cancer [56]. A meta-analysis study further showed longer RALS operating time due to docking and undocking of a robotic cart which has been rectified in da Vinci Xi system [57]. These studies suggest that despite comparable oncologic outcomes by the robotic approach, large and prospective randomized controlled trials are absolutely required to draw a definite conclusion.

Obesity is an important area of interest, and clinically demanding treatment of obese patients. Fung et al. [58] has systematically reviewed that rectal cancer laparoscopic surgery in obese patients is clinically difficult mainly because of longer operating times, higher risk of postoperative complications and higher conversion rates to open surgery. In other case-control retrospective studies, when compared obese versus non-obese patients undergoing robotic colorectal surgery frequently reported insignificant differences in conversion rate, circumferential resection margin rate, intraoperative or postoperative complications, estimated blood loss, operating time and length of hospital stay [59]-[64]. 
In the rectal cancer treatment, surgeons wish to maintain postoperative urinary and sexual function while achieving total tumor resection to retain the quality of life for rectal cancer surgery patients. A few studies addressed the urogenital complications for rectal cancer following RALS to maintain urinary and sexual function after Total Mesorectal Excision (TME) [65]. In several cohort studies, a substantially lower rate of urinary retention following rectal cancer surgery in RALS than conventional laparoscopic surgery was probably due to retention of the neural component with precision [66] [67] [68].

Comparative study on the surgery cost by RALS versus laparoscopic was almost insignificant mainly because of a 50\% less conversion case to open in RALS [69]. Subsequently, the reduced conversion costs and decreased hospital stay might offset the installation and maintenance cost of a new robotic surgical system in the long term.

\subsubsection{Gastric Cancer}

Gastrectomy is the ultimate treatment for gastric cancer. Since Kitano et al. [70] reported the first laparoscopic gastrectomy (LG) followed by the first robotic-assisted gastrectomy (RAG) in 2002, both in Japan, minimally invasive gastrectomy has gained momentum for gastrectomy in gastric cancer patients [71]. The use of RAG has further increased since it was approved in 2008 for national medical insurance coverage in Japan [72]. However, after almost two decades of RAG application for gastric cancer surgery, only limited conclusions can be drawn from several retrospective studies [73]. To date, no randomized clinical trials have been performed comparing RAG, LG or Open Gastrectomy (OG) [74]. However, some cohort studies reported the same oncological outcomes as survival and lymph node yield. The noticeable difference for RAG was reduction in intraoperative blood loss [75] and the risk of pancreatic fistula formation during dissection and D2 lymphadenectomy with RAG compared to LG [74]. However, higher costs and longer operating times are associated with RAG [75]. Overall, the mortality rate and number of harvested lymph nodes for RAG and LG did not differ, but due to less intraoperative blood loss, RAG and LG resulted in a shorter postoperative hospital stay than OG [72] [76] [77]. RAG, however, was associated with longer operating time and higher costs [72]. Although survival data of RAG were like OG, studies on long-term outcomes are needed to confirm these results [78].

\subsubsection{Breast Cancer}

In an esthetic sense, endoscopic breast operation is a need to achieve full cancer clearance while preserving the patient's physique [79]. However, limited maneuverability (due to inflexible endoscopic equipment) and insufficient representation of the operating field (due to 2-dimensional cameras) are disadvantages of this technique [80]. Lately, few studies tried the feasibility and safety of robot-assisted Nipple-sparing Mastectomy (RANSM) followed by immediate breast reconstruction (IBR) to treat breast cancer [79] [80]. It was found that for 
early breast cancer and benign conditions, RANSM along with IBR is surgically safe and feasible. According to a study, RANSM followed by IBR has relatively better results and considerably better patient satisfaction compared to the Conventional Nipple-sparing Mastectomy (CNSM) followed by IBR [81]. The only drawback is being longer operation time and higher cost.

\subsubsection{Prostate Cancer}

The removal of the prostate gland requires extreme surgical precision due to the prostate blood vessels, muscles, and nerve bundles being in very close proximity to each other in addition to the same nerve bundles innervating the erectile and bladder functions resulting in a very delicate structure. Prostatectomy is generally recommended if cancer has not yet spread to the other regions persisting in this area. A robot-assisted procedure is minimally invasive while the traditional open surgery will require an incision from the navel to the pubic bone to access the prostate gland resulting in a huge scar. There are several approaches to prostatectomy, one of which is Radical Prostatectomy (RP) which is being implemented in robotic surgery and named Robot-Assisted Radical Prostatectomy (RARP). Possible negative outcomes of this procedure are erectile dysfunction and Postoperative Prostatectomy Incontinence (PPI) [82]. There are many surgical approaches to implement RARP [83], however not enough evidence to support that these procedures are oncologically safe. The commonly used approaches are Retzius-sparing RARP which has been efficient in earlier recovery from urinary incontinence, a side effect of the surgical treatment of prostate cancer [83] [84]. Nevertheless, RARP is opted by $86 \%$ of prostate cancer patients in the United States [13].

As there has been a lack of well-controlled randomized clinical trials to compare, there is no high-quality evidence to inform the comparative effectiveness of LRP or RARP compared to Open Radical Prostatectomy (ORP) for oncological outcomes. Few meta-analysis studies reported that RARP results in better overall short term outcomes compared to LRP or ORP as it is being oncologically safe, reducing hospital stay, less blood loss, nerve-sparing, as well as earlier recovery from urinary incontinence and erectile dysfunction [85] [86] [87]. Moreover, in an earlier study, in addition to the similar short-term outcomes, they reported no difference in urinary and sexual quality of life in RARP, LRP or ORP, however more evidence and randomized controlled trials are necessary to confirm the safety of this approach [88].

\subsubsection{Gynecological Cancers}

Gynecologic cancers are one of the more prominent cancers for females after breast cancer which includes cancers of ovary, uterine, cervical, vulvar and vaginal. Hysterectomy is one of the widely implemented surgical procedures performed in these cancers depending on the stage of cancer, the localization of the tumor, and which other organs have cancer affected in the pelvic cavity. Surgical treatment for gynecological cancers implementing OS has been the standard ap- 
proach until the introduction of MIS using laparoscopy in 1992 [89] [90] although the first Total Laparoscopic Radical Hysterectomy (TLRH) was performed by Nezhat and colleagues in 1989 [90]. Compared to the OS approach, Laparoscopic Radical Hysterectomy (LRH) proved to have some benefits over the traditional open abdominal approach like lower blood loss, preservation of soft tissues, faster wound healing, less recovery time [91] [92]. To these benefits of LRH, there are inherent ergonomic drawbacks like the posture of the surgeon performing the surgery, the $2 \mathrm{D}$ visuals of the surgical site, and the maneuverability of the laparoscopic instruments [93] [94]. The learning curve for LRH or laparoscopic surgeries, in general, is notably long as it might be difficult for surgeons who are experienced in open surgeries to train expertly in laparoscopic surgery. Moreover, the patient survival and successful procedure rate was almost the same in both methods, cost being the differential factor for patients who opted for LRH [95].

Following Da Vinci surgical system approval by the USFDA, the Robot-Assisted Radical Hysterectomy (RARH) for gynecological surgeries was introduced in 2005 [96] [97]. Da Vinci Xi surgical system was opted by many surgeons due to its superior maneuverability, 3D visualization of the target site, relatively easy learning curve and better ergonomics compared to the laparoscopic methods [14] [98] [99]. Some randomized controlled trials have reported that the RARH is superior to LRH with minor differences recorded in blood loss, operative time, maneuverability, hospital stay, postoperative complications [100] [101] [102].

In addition, the RARH and LRH approaches require small incisions to perform a hysterectomy [103] as compared to the OS approach which requires a large vertical incision from the navel to the pubic bone or a horizontal incision in the lower abdomen to access the uterus, depending on the procedure opted by surgeons. Several recent studies have also found promising results concerning the use of minimally invasive procedures (RARH and LRH) for the treatment of gynecological cancers to be safe and feasible with comparatively less postoperative complications and better quality of life post-surgery [104]-[110].

\section{Advantages and Disadvantages of Da Vinci Surgical System}

Being a man-made machine, da Vinci surgical robot ought to have some advantages as well as disadvantages which are summarized below.

\subsection{Advantages}

\subsubsection{Smaller Incisions and Less Trauma}

The essence of robotic surgery is less invasive which means less discomfort and quicker recovery time for the patient. The surgical arms are often pneumatic to control the operation, powered by compressed air and electricity. The "hands" of each arm, containing each of the necessary surgical instruments, are often 
smaller than human hands, reducing the need for large incisions. Surgeries that normally result in a postoperative hospital stay lasting for a week may potentially become outpatient procedures. For example, a liver resection after a conventional procedure will hold a patient hospital-bound for a week or more. The use of a robot reduces the stay in the hospital to only one or two days.

\subsubsection{Higher Surgical Accuracy}

Many operations-from orthopedic surgery to neurological procedures-need a high degree of precision. One slip of the scalpel or misplaced collection of forceps can injure the patient or even lead to death. In Robot surgery, robots aren't susceptible to a human surgeon's shaking or other strain-related motions. When the hands of the controller shake, the software of the system compensates for the shaking, and this does not affect the operation.

\subsubsection{Reduced Surgeon Fatigue}

Surgeries, particularly those which take many hours, are exhausting for the surgeon in attendance. The staff will be on their feet for hours at a time, finishing the surgery, and taking care of the patient. Surgical robots allow the surgeon to sit comfortably while conducting operations. This could help keep surgeons fresh and conscious for the duration of their surgery, prevent fatigue-related errors, and reduce the potential for medical malpractice.

\subsection{Disadvantages}

\subsubsection{Expense of Surgery}

Surgery is an expensive process at best. The high cost of installing a robotic operating system can increase the cost of surgery. Surgical robots are costly to maintain, and additional training is needed for their service, which is also costly. Precise figures are hard to come by, however, in general, surgery using a da Vinci surgery robot will cost between $\$ 3000$ and $\$ 6000$ more than a traditional laparoscopic procedure.

\subsubsection{Movement Latency}

One of the most important robotic surgery issues is the question of latency-the time it takes for the robot to perform the surgeon's commands. It takes the machine several moments to connect with the robotic arms. Although this is not a regular surgery concern, surgeons find it difficult to respond quickly to problems that arise during the surgery.

\section{Conclusions and Future Perspectives}

Since its introduction in 1985, robotic surgery has been widely used in various fields, due to its several incomparable advantages. High-resolution, 10-fold image magnification and 3-dimensional optics enable delicate structures like intercostal perforators and lymphatics to be accurately visualized and differentiated. The complex and intuitive motion of the robotic arms allows for micro- 
scale manipulation, and even in a small operating area, surgeons can accurately perform delicate tasks. Therefore, robotic surgery scores over laparoscopic surgery and is commonly used in many intracorporeal procedures. However, except the high precision, shorter hospital stays, less intraoperative blood loss, robotic surgery with da Vinci surgical systems are associated with high cost and longer operative time but delivered similar oncological outcomes as laparoscopic surgery. Almost all types of cancer surgeries reviewed here were based on retrospective studies and everyone cited lack of quality randomized controlled trials to compare the robotic versus laparoscopic versus open approaches. Going by the expensive set up and recurring costs, it might be the best option for the Intuitive Surgical (USA) to coordinate multicentered international clinical trials for various cancers to stay afloat in the intense future competition in the robotic surgery market.

Further, it is also a fact that not all the surgical procedures can be performed on da Vinci Surgical Systems, several specific robots are being developed such as PRECEYES Surgical System (Preceyes B.V, The Netherlands) and Versius Surgical Systems (CMR Surgical Ltd, UK). With the advancing technology many more robots aimed towards robot assisted surgery are expected to be developed soon.

\section{Acknowledgements}

Yusuf Jamal is pursuing MBBS degree from TNMU, Ukraine.

\section{Conflicts of Interest}

The authors declare no conflicts of interest regarding the publication of this paper.

\section{References}

[1] Kwoh, Y.S., Hou, J., Jonckheere, E.A. and Hayati, S.A. (1988) Robot with Improved Absolute Positioning Accuracy for CT Guided Stereotactic Brain Surgery. IEEE Transactions on Biomedical Engineering, 35, 153-160. https://doi.org/10.1109/10.1354

[2] Davies, B.L., Hibberd, R.D., Ng, W.S., Timoney, A.G. and Wickham, J.E.A. (1991) The Development of a Surgeon Robot for Prostatectomies. Proceedings of the Institution of Mechanical Engineers, Part H: Journal of Engineering in Medicine, 205 35-38. https://doi.org/10.1243/PIME PROC 199120525902

[3] Bargar, W.L. (2007) Robots in Orthopaedic Surgery: Past, Present, and Future. Clinical Orthopaedics and Related Research, 463, 31-36. https://doi.org/10.1097/BLO.0b013e318146874f

[4] Lane, T. (2018) A Short History of Robotic Surgery. Annals of the Royal College of Surgeons of England, 100, 5-7. https://doi.org/10.1308/rcsann.supp1.5

[5] Satava, R.M. (2002) Surgical Robotics: The Early Chronicles: A Personal Historical Perspective. Surg. Surgical Laparoscopy, Endoscopy \& Percutaneous Techniques, 12, 6-16. https://doi.org/10.1097/00129689-200202000-00002

[6] Bellamy, R.F., Zajtchuk, R., Buescher, T.M., et al. (1991) Part I: Warfare, Weaponry, 
and the Casualty: Conventional Warfare: Ballistic, Blast, and Burn Injuries. Vol. 5, Office of the Surgeon General Department of the Army, Washington DC.

[7] Zajtchuk, R, and Grande, C. (1995) Part IV: Surgical Combat Casualty Care: Anesthesia and Perioperative Care of the Combat Casualty. Vol. 1, Office of the Surgeon General Department of the Army, Washington DC.

[8] Felger, J.E., Nifong, L. and Chitwood, W.R. (2002) The Evolution of an Early Experience with Robot-Assisted Mitral Valve Surgery. Surgical Laparoscopy, Endoscopy \& Percutaneous Techniques, 12, 58-63. https://doi.org/10.1097/00129689-200202000-00010

[9] George, E.I., Brand, T.C., La Porta, A., Marescaux, J. and Satava, R.M. (2018) Origins of Robotic Surgery: From Skepticism to Standard of Care. Journal of the Society of Laparoscopic \& Robotic Surgeons, 22, e2018. https://dx.doi.org/10.4293\%2FJSLS.2018.00039

[10] Ranev, D. and Teixeira, J. (2020) History of Computer-Assisted Surgery. Surgical Clinics of North America, 100, 209-218. https://doi.org/10.1016/j.suc.2019.11.001

[11] Madhani, A.J., Niemeyer, G. and Salisbury, J.K. (1998) The Black Falcon: A Teleoperated Surgical Instrument for Minimally Invasive Surgery. IEEE/ RSJ International Conference on Intelligent Robots and Systems. Innovations in Theory, Practice and Applications, Vol. 2, Victoria, 17 October 1998, 936-944.

[12] Salisbury Jr., J.K. (1995) Look and Feel: Haptic Interaction for Biomedicine. Massachusetts Institute of Technology Cambridge Artificial Intelligence Lab, Massachusetts.

[13] Crew, B. (2020) Worth the Cost? A Closer Look at the da Vinci Robot's Impact on Prostate Cancer Surgery. Nature, 580, S5-S7. https://doi.org/10.1038/d41586-020-01037-w

[14] Gala, R.B., Margulies, R., Steinberg, A., Murphy, M., Lukban, J., Jeppson, P., et al. (2014) Systematic Review of Robotic Surgery in Gynecology: Robotic Techniques Compared with Laparoscopy and Laparotomy. Journal of Minimally Invasive Gynecology, 21, 353-361. https://doi.org/10.1016/j.jmig.2013.11.010

[15] Intuitive Surgical Inc. (2001) United States Securities and Exchange Commission Form 10-K. Intuitive Surgical Inc., Sunnyvale.

[16] Demmy, T.L and Curtis, J.J. (1999) Minimally Invasive Lobectomy Directed toward Frail and High-Risk Patients a Case-Control Study. The Annals of Thoracic Surgery, 68, 194-200. https://doi.org/10.1016/S0003-4975(99)00467-1

[17] Nakata, M., Saeki, H., Yokoyama, N., Kurita, A., Takiyama, W. and Takashima, S. (2000) Pulmonary Function after Lobectomy Video-Assisted Thoracic Surgery versus Thoracotomy. The Annals of Thoracic Surgery, 70, 938-941. https://doi.org/10.1016/S0003-4975(00)01513-7

[18] Yim, A.P., Wan, S., Lee, T.W. and Arifi, A.A. (2000) VATS Lobectomy Reduces Cytokine Responses Compared with Conventional Surgery. The Annals of Thoracic Surgery, 70, 243-247. https://doi.org/10.1016/S0003-4975(00)01258-3

[19] Hoksch, B., Ablassmaier, B., Walter, M. and Muller, J.M. (2003) Complication Rate after Thoracoscopic and Conventional Lobectomy. Zentralblatt Fur Chirurgie, 128, 106-110. https://doi.org/10.1055/s-2003-37763

[20] Li, W.W., Lee, R.L., Lee, T.W., Ng, C.S., Sihoe, A.D., Wan, I.Y., et al. (2003) The Impact of Thoracic Surgical Access on Early Shoulder Function: Video-Assisted Thoracic Surgery versus Posterolateral Thoracotomy. European Journal of Cardio- Thoracic Surgery, 23, 390-396. https://doi.org/10.1016/s1010-7940(02)00795-9 
[21] Nomori, H., Ohtsuka, T., Horio, H., Naruke, T. and Suemasu, K. (2003) Difference in the Impairment of Vital Capacity and 6-Minute Walking after a Lobectomy Performed by Thoracoscopic Surgery, an Anterior Limited Thoracotomy, an Anteroaxillary Thoracotomy, and a Posterolateral Thoracotomy. Surgery Today, 33, 7-12. https://doi.org/10.1007/s005950300001

[22] Seder, C.W., Salati, M., Kozower, B.D., Wright, C.D., Falcoz, P.E., Brunelli, A., et al. (2016) Variation in Pulmonary Resection Practices between the Society of Thoracic Surgeons and the European Society of Thoracic Surgeons General Thoracic Surgery Databases. The Annals of Thoracic Surgery, 101, 2077-2084. https://doi.org/10.1016/j.athoracsur.2015.12.073

[23] McKenna Jr., R.J., Wolf, R.K., Brenner, M., Fischel, R.J. and Wurnig, P. (1998) Is Lobectomy by Video-Assisted Thoracic Surgery an Adequate Cancer Operation? The Annals of Thoracic Surgery, 66, 1903-1908. https://doi.org/10.1016/S0003-4975(98)01166-7

[24] Leschber, G. and Holinka, G. and Linder, A. (2003) Video-Assisted Mediastinoscopic Lymphadenectomy (VAMLA) - A Method for Systematic Mediastinal Lymphnode Dissection. European Journal of Cardio-Thoracic Surgery, 24, 192-195. https://doi.org/10.1016/S1010-7940(03)00253-7

[25] Park, B.J., Flores, R.M. and Rusch, V.W. (2006) Robotic Assistance for Video-Assisted Thoracic Surgical Lobectomy: Technique and Initial Results. The Journal of Thoracic and Cardiovascular Surgery, 131, 54-59.

https://doi.org/10.1016/j.jtcvs.2005.07.031

[26] Melfi, F.M. and Mussi, A. (2008) Robotically Assisted Lobectomy: Learning Curve and Complications. Thoracic Surgery Clinics, 18, 289-295.

https://doi.org/10.1016/j.thorsurg.2008.06.001

[27] Giulianotti, P.C., Buchs, N.C., Caravaglios, G. and Bianco, F.M. (2010) Robot-Assisted Lung Resection: Outcomes and Technical Details. Interactive Cardio Vascular and Thoracic Surgery, 11, 388-392.

https://doi.org/10.1510/icvts.2010.239541

[28] Veronesi, G., Galetta, D., Maisonneuve, P., Melfi, F., Schmid, R.A., Borri, A., et al. (2010) Four-Arm Robotic Lobectomy for the Treatment of Early-Stage Lung Cancer. The Journal of Thoracic and Cardiovascular Surgery, 140, 19-25. https://doi.org/10.1016/j.jtcvs.2009.10.025

[29] Cerfolio, R.J., Bryant, A.S., Skylizard, L. and Minnich, D.J. (2011) Initial Consecutive Experience of Completely Portal Robotic Pulmonary Resection with 4 Arms. The Journal of Thoracic and Cardiovascular Surgery, 142, 740-746. https://doi.org/10.1016/j.jtcvs.2011.07.022

[30] Dylewski, M.R., Ohaeto, A.C. and Pereira, J.F. (2011) Pulmonary Resection Using a Total Endoscopic Robotic Video-Assisted Approach. Seminars in Thoracic and Cardiovascular Surgery, 23, 36-42. https://doi.org/10.1053/j.semtcvs.2011.01.005

[31] Louie, B.E., Farivar, A.S., Aye, R.W. and Vallières, E. (2012) Early Experience with Robotic Lung Resection Results in Similar Operative Outcomes and Morbidity When Compared with Matched Video-Assisted Thoracoscopic Surgery Cases. The Annals of Thoracic Surgery, 93, 1598-1605.

https://doi.org/10.1016/j.athoracsur.2012.01.067

[32] Abbas, A.E. (2018) Surgical Management of Lung Cancer: History, Evolution, and Modern Advances. Current Oncology Reports, 20, Article No. 98. https://doi.org/10.1007/s11912-018-0741-7

[33] Park, B.J., Melfi, F., Mussi, A., Maisonneuve, P., Spaggiari, L., Da Silva, R.K., et al. (2012) Robotic Lobectomy for Non-Small Cell Lung Cancer (NSCLC): Long-Term 
Oncologic Results. The Journal of Thoracic and Cardiovascular Surgery, 143, 383-389. https://doi.org/10.1016/j.jtcvs.2011.10.055

[34] Muhe, E. (1992) Long-Term Follow-Up after Laparoscopic Cholecystectomy. Endoscopy, 24, 754-758. https://doi.org/10.1055/s-2007-1009119

[35] Litynski, G.S. (1999) Mouret, Dubois, and Perissat: The Laparoscopic Breakthrough in Europe (1987-1988). Journal of the Society of Laparoscopic \& Robotic Surgeons, 3, 163-167.

[36] Hu, L.D., Yao, L., Li, X.F., Jin, P.H., Yang, K.H. and Guo, T.K. (2018) Effectiveness and Safety of Robotic-Assisted versus Laparoscopic Hepatectomy for Liver Neoplasms: A Meta-Analysis of Retrospective Studies. Asian Journal of Surgery, 41, 401-416. https://doi.org/10.1016/j.asjsur.2017.07.001

[37] Fahrner, R., Rauchfuß, F., Bauschke, A., Kissler, H., Settmacher, U. and Zanow, J. (2019) Robotic Hepatic Surgery in Malignancy: Review of the Current Literature. Journal of Robotic Surgery, 13, 533-538. https://doi.org/10.1007/s11701-019-00939-w

[38] Lafaro, K.J., Stewart, C., Fong, A. and Fong, Y. (2020) Robotic Liver Resection. Surgical Clinics of North America, 100, 265-281. https://doi.org/10.1016/j.suc.2019.11.003

[39] Tsilimigras, D.I., Moris, D., Vagios, S., Merath, K. and Pawlik, T.M. (2018) Safety and Oncologic Outcomes of Robotic Liver Resections: A Systematic Review. Journal of Surgical Oncology, 117, 1517-1530. https://doi.org/10.1002/jso.25018

[40] Melstrom, L.G., Warner, S.G., Woo, Y., Sun, V., Lee, B., Singh, G. and Fong, Y. (2018) Selecting Incision-Dominant Cases for Robotic Liver Resection: Towards Outpatient Hepatectomy with Rapid Recovery. Hepatobiliary Surgery and Nutrition, 7, 77-84. https://doi.org/10.21037/hbsn.2017.05.05

[41] Nota, C.L., Woo, Y., Raoof, M., Boerner, T., Molenaar, I.Q., Choi, G.H., et al. (2019) Robotic versus Open Minor Liver Resections of the Posterosuperior Segments: A Multinational, Propensity Score-Matched Study. Annals of Surgical Oncology, 26, 583-590. https://doi.org/10.1245/s10434-018-6928-1

[42] Khan, S., Beard, R.E., Kingham, P.T., Fong, Y., Boerner, T., Martinie, J.B., et al. (2018) Long-Term Oncologic Outcomes following Robotic Liver Resections for Primary Hepatobiliary Malignancies: A Multicenter Study. Annals of Surgical Oncology, 25, 2652-2660. https://doi.org/10.1245/s10434-018-6629-9

[43] Guan, R.Y., Chen, Y.J., Yang, K., Ma, D., Gong, X.Y., Shen, B.Y., et al. (2019) Clinical Efficacy of Robot-Assisted versus Laparoscopic Liver Resection: A Meta-Analysis. Asian Journal of Surgery, 42, 19-31. https://doi.org/10.1016/j.asjsur.2018.05.008

[44] Fruscione, M., Pickens, R., Baker, E.H., Cochran, A., Khan, A., Ocuin, L., et al. (2019) Robotic-Assisted versus Laparoscopic Major Liver Resection: Analysis of Outcomes from a Single Center. $H P B($ Oxford $), 21,906-911$. https://doi.org/10.1016/j.hpb.2018.11.011

[45] Cooperman, A., Katz, V., Zimmon, D. and Botero, G. (1991) Laparoscopic Colon resection: A Case Report. Journal of Laparoendoscopic Surgery, 1, 221-224. https://doi.org/10.1089/lps.1991.1.221

[46] Jacobs, M., Verdeja, J. and Goldstein, H. (1991) Minimally Invasive Colon Resection (Laparoscopic Colectomy). Surgical Laparoscopy Endoscopy \& Percutaneous Techniques, 1, 144-150.

[47] Fowler, D. and White, S. (1991) Laparoscopy-Assisted Sigmoid Resection. Surgical 
Laparoscopy Endoscopy \& Percutaneous Techniques, 1, 183-188.

[48] Lacy, A.M., García-Valdecasas, J.C., Delgado, S., Castells, A., Taurá, P., Piqué, J.M., et al. (2002) Laparoscopy-Assisted Colectomy versus Open Colectomy for Treatment of Non-Metastatic Colon Cancer: A Randomized Trial. Lancet, 359, 2224-2229. https://doi.org/10.1016/S0140-6736(02)09290-5

[49] Abraham, N.S., Young, J.M. and Solomon, M.J. (2004) Meta-Analysis of Short-Term Outcomes after Laparoscopic Resection for Colorectal Cancer. British Journal of Surgery, 91, 1111-1124. https://doi.org/10.1002/bjs.4640

[50] Braga, M., Frasson, M., Zuliani, W., Vignali, A., Pecorelli, N. and Di Carlo, V. (2010) Randomized Clinical Trial of Laparoscopic versus Open Left Colonic Resection. British Journal of Surgery, 97, 1180-1186. https://doi.org/10.1002/bjs.7094

[51] Marusch, F., Gastinger, I., Schneider, C., Scheidbach, H., Konradt, J., Bruch, H.P., et al. (2001) Importance of Conversion for Results Obtained with Laparoscopic Colorectal Surgery. Diseases of the Colon \& Rectum, 44, 207-214. https://doi.org/10.1007/BF02234294

[52] Hewett, P.J., Allardyce, R.A., Bagshaw, P.F., Frampton, C.M., Frizelle, F.A., Rieger, N.A., et al. (2008) Short-Term Outcomes of the Austral-Asian Randomized Clinical Study Comparing Laparoscopic and Conventional Open Surgical Treatments for Colon Cancer: The ALCCaS Trial. Annals of Surgery, 248, 728-738.

https://doi.org/10.1097/SLA.0b013e31818b7595

[53] Bagshaw, P.F., Allardyce, R.A., Frampton, C.M., Frizelle, F.A., Hewett, P.J., McMurrick, P.J., et al, (2012) Long-Term Outcomes of the Australasian Randomized Clinical Trial Comparing Laparoscopic and Conventional Open Surgical Treatments for Colon Cancer: The Australasian Laparoscopic Colon Cancer Study Trial. Annals of Surgery, 256, 915-919. https://doi.org/10.1097/SLA.0b013e3182765ff8

[54] McCombie, A.M., Frizelle, F., Bagshaw, P.F., Frampton, C.M., Hewett, P.J., McMurrick, P.J., et al. (2018) The ALCCaS Trial: A Randomized Controlled Trial Comparing Quality of Life Following Laparoscopic versus Open Colectomy for Colon Cancer. Diseases of the Colon \& Rectum, 61, 1156-1162. https://doi.org/10.1097/DCR.0000000000001165

[55] Polat, F., Willems, L.H., Dogan, K. and Rosman, C. (2019) The Oncological and Surgical Safety of Robot-Assisted Surgery in Colorectal Cancer: Outcomes of a Longitudinal Prospective Cohort Study. Surgical Endoscopy, 33, 3644-3655. https://doi.org/10.1007/s00464-018-06653-2

[56] Kang, J., Park, Y.A., Baik, S.H., Sohn, S.K. and Lee, K.Y. (2016) A Comparison of Open, Laparoscopic, and Robotic Surgery in the Treatment of Right-Sided Colon Cancer. Surgical Laparoscopy, Endoscopy \& Percutaneous Techniques, 26, 497-502. https://doi.org/10.1097/SLE.0000000000000331

[57] Matsuyama, T., Kinugasa, Y., Nakajima, Y. and Kojima, K. (2018) Robotic-Assisted Surgery for Rectal Cancer: Current State and Future Perspective. Annals of Gastroenterological Surgery, 2, 406-412. https://doi.org/10.1002/ags3.12202

[58] Fung, A., Trabulsi, N., Morris, M., Garfinkle, R., Saleem, A., Wexner, S.D., et al. (2017) Laparoscopic Colorectal Cancer Resections in the Obese: A Systematic Review. Surgical Endoscopy, 31, 2072-2088.

https://doi.org/10.1007/s00464-016-5209-y

[59] Hellan, M., Ouellette, J., Lagares-Garcia, J.A., Rauh, S.M. and Kennedy, H.L., Nicholson, J.D., et al. (2015) Robotic Rectal Cancer Resection: A Retrospective Multicenter Analysis. Annals of Surgical Oncology, 22, 2151-2158.

https://doi.org/10.1245/s10434-014-4278-1 
[60] Gorgun, E., Ozben, V., Costedio, M., Stocchi, L., Kalady, M. and Remzi, F. (2016) Robotic versus Conventional Laparoscopic Rectal Cancer Surgery in Obese Patients. Colorectal Disease, 18, 1063-1071. https://doi.org/10.1111/codi.13374

[61] Shiomi, A., Kinugasa, Y., Yamaguchi, T., Kagawa, H. and Yamakawa, Y. (2016) Robot-Assisted versus Laparoscopic Surgery for Lower Rectal Cancer: The Impact of Visceral Obesity on Surgical Outcomes. International Journal of Colorectal Disease, 31, 1701-1710. https://doi.org/10.1007/s00384-016-2653-Z

[62] Baukloh, J.K., Reeh, M., Spinoglio, G., Corratti, A., Bartolini, I., Mirasolo, V.M., et al. (2017) Evaluation of the Robotic Approach Concerning Pitfalls in Rectal Surgery. European Journal of Surgical Oncology, 43, 1304-1311. https://doi.org/10.1016/j.ejso.2016.12.014

[63] Bayraktar, O., Aytac, E., Ozben, V., Atasoy, D., Bilgin, İ.A., Bayraktar, İ.E., et al. (2018) Does Robot Overcome Obesity-Related Limitations of Minimally Invasive Rectal Surgery for Cancer? Surgical Laparoscopy, Endoscopy \& Percutaneous Techniques, 28, e8-e11. https://doi.org/10.1097/SLE.0000000000000500

[64] Panteleimonitis, S., Pickering, O., Abbas, H., Harper, M., Kandala, N., Figueiredo, N., et al. (2018) Robotic Rectal Cancer Surgery in Obese Patients May Lead to Better Short-Term Outcomes When Compared to Laparoscopy: A Comparative Propensity Scored Match Study. International Journal of Colorectal Disease, 33, 1079-1086. https://doi.org/10.1007/s00384-018-3030-x

[65] Luca, F., Valvo, M., Ghezzi, T.L., Zuccaro, M., Cenciarelli, S., Trovato, C., et al. (2013) Impact of Robotic Surgery on Sexual and Urinary Functions after Fully Robotic Nerve-Sparing Total Mesorectal Excision for Rectal Cancer. Annals of Surgery, 257, 672-678. https://doi.org/10.1097/SLA.0b013e318269d03b

[66] Cho, M.S., Baek, S.J., Hur, H., Min, B.S., Baik, S.H., Lee, K.Y., et al. (2015) Short and long-Term Outcomes of Robotic versus Laparoscopic Total Mesorectal Excision for Rectal Cancer: A Case-Matched Retrospective Study. Medicine, 94, e522. https://doi.org/10.1097/MD.0000000000000522

[67] Yamaguchi, T., Kinugasa, Y., Shiomi, A., Tomioka, H., Kagawa, H. and Yamakawa, Y. (2016) Robotic-Assisted vs. Conventional Laparoscopic Surgery for Rectal Cancer: Short-Term Outcomes at a Single Center. Surgery Today, 46, 957-962. https://doi.org/10.1007/s00595-015-1266-4

[68] Law, W.L. and Foo, D.C.C. (2017) Comparison of short-Term and Oncologic Outcomes of Robotic and Laparoscopic Resection for Mid- and Distal Rectal Cancer. Surgical Endoscopy, 31, 2798-2807. https://doi.org/10.1007/s00464-016-5289-8

[69] Cleary, R.K., Mullard, A.J., Ferraro, J. and Regenbogen, S.E. (2018) The Cost of Conversion in Robotic and Laparoscopic Colorectal Surgery. Surgical Endoscopy, 32, 1515-1524. https://doi.org/10.1007/s00464-017-5839-8

[70] Kitano, S., Iso, Y., Moriyama, M. and Sugimachi, K. (1994) Laparoscopy Assisted Billroth I Gastrectomy. Surgical Laparoscopy Endoscopy \& Percutaneous Techniques, 4, 146-148.

[71] Hashizume, M., Shimada, M., Tomikawa, M., Ikeda, Y., Takahashi, I., Abe, R., et al. (2002) Early Experiences of Endoscopic Procedures in General Surgery Assisted by a Computer Enhanced Surgical System. Surgical Endoscopy and other Interventional Techniques, 16, 1187-1191. https://doi.org/10.1007/s004640080154

[72] Shibasaki, S., Suda, K., Obama, K., Yoshida, M. and Uyama, I. (2020) Should Robotic Gastrectomy Become a Standard Surgical Treatment Option for Gastric Cancer? Surgery Today, 50, 955-965. https://doi.org/10.1007/s00595-019-01875-w

[73] Russo, A.E. and Strong, V.E. (2019) Gastric Cancer Etiology and Management in Asia and the West. Annual Review of Medicine, 70, 353-367. 
https://doi.org/10.1146/annurev-med-081117-043436

[74] Van Boxel, G.I., Ruurda, J.P. and van Hillegersberg, R. (2019) Robotic-Assisted Gastrectomy for Gastric Cancer: A European Perspective. Gastric Cancer, 22, 909-919. https://doi.org/10.1007/s10120-019-00979-Z

[75] Zheng, B., Wang, X., Li, J., Wang, Q., Bi, L., Deng, X.B. and Wang, Z.Q. (2019) Robotic Gastrectomy versus Laparoscopic Gastrectomy for Gastric Cancer: Meta-Analysis and Trial Sequential Analysis of Prospective Observational Studies. Surgical Endoscopy, 33, 1033-1048. https://doi.org/10.1007/s00464-018-06648-Z

[76] Shen, W.S., Xi, H.Q., Chen, L. and Wei, B. (2014) A Meta-Analysis of Robotic versus Laparoscopic Gastrectomy for Gastric Cancer. Surgical Endoscopy, 28, 2795-2802. https://doi.org/10.1007/s00464-014-3547-1

[77] Kim, H.I., Han, S.U., Yang, H.K., Kim, Y.W., Lee, H.J., Ryu, K.W., et al. (2016) Multicenter Prospective Comparative Study of Robotic versus Laparoscopic Gastrectomy for Gastric Adenocarcinoma. Annals of Surgery, 263, 103-109. https://doi.org/10.1097/SLA.0000000000001249

[78] Solaini, L., Avanzolini, A., Pacilio, C.A., Cucchetti, A., Cavaliere, D. and Ercolani, G. (2020) Robotic Surgery for Gastric Cancer in the West: A Systematic Review and Meta-Analyses of Short- and Long-Term Outcomes. International Journal of Surgery, 83, 170-175. https://doi.org/10.1016/j.ijsu.2020.08.055

[79] Leff, D.R., Vashisht, R., Yongue, G., et al (2011) Endoscopic Breast Surgery: Where Are We Now and What Might the Future Hold for Video-Assisted Breast Surgery? Breast Cancer Research and Treatment, 125, 607-625. https://doi.org/10.1007/s10549-010-1258-4

[80] Toesca, A., Peradze, N., Manconi, A., Galimberti, V., Intra, M., Colleoni, M., et al. (2017) Robotic Nipple-Sparing Mastectomy for the Treatment of Breast Cancer: Feasibility and Safety Study. Breast, 31, 51-56. https://doi.org/10.1016/j.breast.2016.10.009

[81] Lai, H.W., Chen, S.T., Mok, C.W., Lin, Y.J., Wu, H.K., Lin, S.L., et al. (2020) Robotic versus Conventional Nipple Sparing Mastectomy and Immediate Gel Implant Breast Reconstruction in the Management of Breast Cancer-A Case Control Comparison Study with Analysis of Clinical Outcome, Medical Cost, and $\mathrm{Pa}$ tient-Reported Cosmetic Results. Journal of Plastic, Reconstructive \& Aesthetic Surgery, 73, 1514-1525. https://doi.org/10.1016/j.bjps.2020.02.021

[82] Mottet, N., Bellmunt, J., Bolla, M., Briers, E., Cumberbatch, M.G., De Santis, M., et al (2017) EAU-ESTRO-SIOG Guidelines on Prostate Cancer. Part 1: Screening, Diagnosis, and Local Treatment with Curative Intent. European Urology, 71, 618-629. https://doi.org/10.1016/j.eururo.2016.08.003

[83] Martini, A., Falagario, U.G., Villers, A., Dell'Oglio, P., Mazzone, E., Autorino, R., et al. (2020) Contemporary Techniques of Prostate Dissection for Robot-Assisted Prostatectomy. European Urology, 78, 583-591. https://doi.org/10.1016/j.eururo.2020.07.017

[84] Collette, E., Klaver, S., Lissenberg-Witte, B., van den Ouden, D., van Moorselaar, R. and Vis, A. (2020) Patient Reported Outcome Measures Concerning Urinary Incontinence after Robot Assisted Radical Prostatectomy: Development and Validation of an Online Prediction Model Using Clinical Parameters, Lower Urinary Tract Symptoms and Surgical Experience. Journal of Robotic Surgery, in press. https://doi.org/10.1007/s11701-020-01145-9

[85] Du, Y.F., Long, Q.Z., Guan, B., Mu, L.J., Tian, J.H., Jiang, Y.M., et al. (2018) Robot-Assisted Radical Prostatectomy Is More Beneficial for Prostate Cancer Patients: A System Review and Meta-Analysis. Medical Science Monitor, 24, 272-287. 
https://doi.org/10.12659/MSM.907092

[86] Theisen, K.M., Myrga, J.M., Hale, N., Cochran, G., Sewall, C., Macleod, L.C., et al. (2019) Excessive Opioid Prescribing after Major Urologic Procedures. Urology, 123, 101-107. https://doi.org/10.1016/j.urology.2018.06.057

[87] Mazzone, E., Dell’Oglio, P., Rosiello, G., Puliatti, S., Brook, N., Turri, F., et al. (2020) Technical Refinements in Superextended Robot-Assisted Radical Prostatectomy for Locally Advanced Prostate Cancer Patients at Multiparametric Magnetic Resonance Imaging. European Urology, in press. https://doi.org/10.1016/j.eururo.2020.09.009

[88] Ilic, D., Evans, S.M., Allan, C.A., Jung, J.H., Murphy, D. and Frydenberg, M. (2017) Laparoscopic and Robotic-Assisted versus Open Radical Prostatectomy for the Treatment of Localised Prostate Cancer. Cochrane Database of Systematic Reviews, 9, Article ID: CD009625. https://doi.org/10.1002/14651858.CD009625.pub2

[89] Canis, M., Maze, G., Wattiez, A., Pouly, J., Chapron, C. and Bruhat, M. (1992) Vaginally Assisted Laparoscopic Radical Hysterectomy. Journal of Gynecologic Surgery, 8, 103-105. https://doi.org/10.1089/gyn.1992.8.103

[90] Nezhat, C.R., Burrell, M.O., Nezhat, F.R., Benigno, B.B. and Welander, C.E. (1992) Laparoscopic Radical Hysterectomy with Paraaortic and Pelvic Node Dissection. American Journal of Obstetrics \& Gynecology, 166, 864-865. https://doi.org/10.1016/0002-9378(92)91351-A

[91] Sharma, R., Bailey, J., Anderson, R. and Murdoch, J. (2006) Laparoscopically Assisted Radical Vaginal Hysterectomy (Coelio-Schauta): A Comparison with Open Wertheim/Meigs Hysterectomy. International Journal of Gynecological Cancer, 16, 1927-1932.

[92] Renato, S., Mohamed, M., Serena, S., Giulia, M., Giulia, F., Giulia, G., et al. (2011) Robot-Assisted Radical Hysterectomy for Cervical Cancer: Review of Surgical and Oncological Outcomes. International Scholarly Research Notices, 2011, Article ID: 872434.

[93] Berguer, R. (1999) Surgery and Ergonomics. The Archives of Surgery, 134, 1011-1016. https://doi.org/10.1001/archsurg.134.9.1011

[94] Dakin, G.F. and Gagner, M. (2003) Comparison of Laparoscopic Skills Performance between Standard Instruments and Two Surgical Robotic Systems. Surgical Endoscopy and other Interventional Techniques, 17, 574-579. https://doi.org/10.1007/s00464-002-8938-Z

[95] Zakashansky, K., Chuang, L., Gretz, H., Nagarsheth, N.P., Rahaman, J. and Nezhat, F.R. (2007) A Case-Controlled Study of Total Laparoscopic Radical Hysterectomy with Pelvic Lymphadenectomy versus Radical Abdominal Hysterectomy in a Fellowship Training Program. International Journal of Gynecological Cancer, 17, 1075-1082.

[96] Advincula, A.P. and Wang, K. (2009) Evolving Role and Current State of Robotics in Minimally Invasive Gynecologic Surgery. Journal of Minimally Invasive Gynecology, 16, 291-301. https://doi.org/10.1016/j.jmig.2009.03.003

[97] Holloway, R.W., Patel, S.D. and Ahmad, S. (2009) Robotic Surgery in Gynecology. Scandinavian Journal of Surgery, 98, 96-109.

https://doi.org/10.1177/145749690909800205

[98] Nezhat, F.R., Datta, M.S., Liu, C., Chuang, L. and Zakashansky, K. (2008) Robotic Radical Hysterectomy versus Total Laparoscopic Radical Hysterectomy with Pelvic Lymphadenectomy for Treatment of Early Cervical Cancer. Journal of the Society of Laparoscopic \& Robotic Surgeons, 12, 227-237. 
[99] Hassan, S.O., Dudhia, J., Syed, L.H., Patel, K., Farshidpour, M., Cunningham, S.C., et al. (2015) Conventional Laparoscopic vs Robotic Training: Which Is Better for Naive Users? A Randomized Prospective Crossover Study. Journal of Surgical Education, 72, 592-599. https://doi.org/10.1016/j.jsurg.2014.12.008

[100] Netter, A., Jauffret, C., Brun, C., Sabiani, L., Blache, G., Houvenaeghel, G., et al. (2020) Choosing the Most Appropriate Minimally Invasive Approach to Treat Gynecologic Cancers in the Context of an Enhanced Recovery Program: Insights from a Comprehensive Cancer Center. PLoS ONE, 15, e0231793. https://doi.org/10.1371/journal.pone.0231793

[101] Luo, C., Liu, M. and Li, X.L. (2018) Efficacy and Safety Outcomes of Robotic Radical Hysterectomy in Chinese Older Women with Cervical Cancer Compared with Laparoscopic Radical Hysterectomy. BMC Women's Health, 18, Article No. 61. https://doi.org/10.1186/s12905-018-0544-x

[102] Mäenpää, M.M., Nieminen, K., Tomás, E.I., Laurila, M., Luukkaala, T.H. and Mäenpää, J.U. (2016) Robotic-Assisted vs Traditional Laparoscopic Surgery for Endometrial Cancer: A Randomized Controlled Trial. American Journal of Obstetrics and Gynecology, 215, 588.e1-588.e7. https://doi.org/10.1016/j.ajog.2016.06.005

[103] Nitecki, R., Ramirez, P.T., Frumovitz, M., Krause, K.J., Tergas, A.I., Wright, J.D., et al. (2020) Survival after Minimally Invasive vs Open Radical Hysterectomy for Early-Stage Cervical Cancer: A Systematic Review and Meta-Analysis. JAMA Oncology, 6, 1019-1027. https://doi.org/10.1001/jamaoncol.2020.1694

[104] Salehi, S., Brandberg, Y., Åvall-Lundqvist, E., Suzuki, C., Johansson, H., Legerstam, B., et al. (2018) Long-Term Quality of Life after Comprehensive Surgical Staging of High-Risk Endometrial Cancer-Results from the RASHEC Trial. Acta Oncologica, 57, 1671-1676. https://doi.org/10.1080/0284186X.2018.1521987

[105] Giannini, A., Russo, E., Mannella, P., Palla, G., Pisaneschi, S., Cecchi, E., et al. (2017) First Series of Total Robotic Hysterectomy (TRH) Using New Integrated Table Motion for the da Vinci Xi: Feasibility, Safety and Efficacy. Surgical Endoscopy, 31, 3405-3410. https://doi.org/10.1007/s00464-016-5331-x

[106] Abel, M.K., Chan, J.K., Chow, S., Darcy, K., Tian, C., Kapp, D.S., et al. (2020) Trends and Survival Outcomes of Robotic, Laparoscopic, and Open Surgery for Stage II Uterine Cancer. International Journal of Gynecologic Cancer, 30, 1347-1355. https://doi.org/10.1136/ijgc-2020-001646

[107] Chang, W.F., Luo, A.J., Yuan, Y.F., Chen, Y., Xin, Z.R. and Xu, S.S. (2020) Perioperative Complications and Safety Evaluation of Robot-Assisted Radical Hysterectomy of Cervical Cancer after Neoadjuvant Chemotherapy. Cancer Management and Research, 12, 4483-4492. https://doi.org/10.2147/CMAR.S243986

[108] El-Achi, V., Weishaupt, J., Carter, J. and Saidi, S. (2020) Robotic versus Laparoscopic Hysterectomy in Morbidly Obese Women for Endometrial Cancer. Journal of Robotic Surgery, in press. https://doi.org/10.1007/s11701-020-01133-Z

[109] Mereu, L., Berlanda, V., Surico, D., Gardella, B., Pertile, R., Spinillo, A., et al. (2020) Evaluation of Quality of Life, Body Image and Surgical Outcomes of Robotic Total Laparoscopic Hysterectomy and Sentinel Lymph Node Mapping in Low-Risk Endometrial Cancer Patients-A Robotic Gyne Club study. Acta Obstetricia et Gynecologica Scandinavica, 99, 1238-1245. https://doi.org/10.1111/aogs.13844

[110] Nasioudis, D., Frey, M.K., Chapman-Davis, E., Caputo, T.A. and Holcomb, K. (2020) Outcomes of Minimally Invasive Surgery for Patients with Endometrial Carcinoma Involving the Cervix. International Journal of Gynecologic Cancer, 30, 619-625. https://doi.org/10.1136/ijgc-2019-001023 